\title{
Research Progress and Trend of Bilingual Education: Visualization Analysis of SSCI Papers Based on WOS Database
}

\author{
Huiqing $\mathrm{Ma}^{1,}$, J Jing $\mathrm{Lu}^{1, \mathrm{~b}}$ \\ 1School of ethnic education, Shaanxi Normal University, Xian 710062, China. \\ a18729052667@163.com, b920239871@qq.com
}

\begin{abstract}
Based on method for visualization of knowledge graph, visual analysis is carried out on the SSCI thesis of WOS database with the theme of bilingual education. The annual quantities of research papers, high-yield institutions, high-yield scholars, academic influence of scholars, and high-yield journals were analyzed. Secondly, through keyword co-occurrence and clustering, the hot topics of bilingual education research focus on the study of cross-linguistic, preschool children, cognitive reserve, two-way bilingual immersion, content and language integrated learning, comprehensive education, South Africa research, second language, teaching language, first and second language education, teacher identification, and immigrant student. The study of crosslinguistic has become a hot spot. At last, the research ecology of bilingual education is summarized from four aspects of research value, hot topics, research focus and research method, and the trend and direction of the future development in this field are given to some extent.
\end{abstract}

Keywords: Bilingual education, SSCI, cross-linguistic, second language.

\section{Study Objects and Methods}

The Cite space is a kind of visual analysis software which is developed in the background of Scientific Metrology and data visualization, which focuses on the potential knowledge contained in the analysis of scientific analysis. As a result of visualizing the structure, rule and distribution of scientific knowledge, the visualized graph obtained by this method is called "the map of scientific knowledge". The paper retrieved 1845 SSCI papers between 2007 and 2017 with the theme of bilingual education based on WOS database. Based on the map of knowledge visualization method, the research trend and development direction in this field are reviewed through the statistics of the SSCI periodicals. Through keyword co-occurrence analysis, the research hotspots in different periods are reflected. Through the analysis of high production institutions and authors, we can further understand the subject of research in this field and show representative institutions and scholars, so as to understand their research methods and results and the direction of future research, and to reveal the hot spots in the research of bilingual education.

All the data used in this paper are derived from the Web of Science core collection and we retrieved 1845 SSCI papers from 2007 to 2017 with the theme of bilingual education. Starting Citespace5.1, the time span is 2007 - 2017, the time partition is 1 year. Then keywords, Author, Institution, Cited Reference, Cited journal, Cited Author are selected as the analysis objects, and the threshold of time slice is set to 50. In this way, we can start the keyword co-occurrence analysis of objects, analyze the co-citation of institutions, authors and periodicals, and draw corresponding scientific knowledge maps.

\section{Visual Analyses of Research Results}

\subsection{The Annual Quantities Analysis of Academic Articles}

Before analyzing key words, institutions, authors and periodicals, we first focus on the overall development trend of the article. From the chart, we can see that from 2007 to 2012, the annual volume of papers increased over time, while the number of papers in the 2013 years dropped somewhat. From 2014 to 2017, the amount of papers is increasing rapidly, of which 2014 to 2016 is particularly significant. It can be said to be the outbreak period of bilingual education research and 
active development in 2017. In general, the study on bilingual education is on the rise and has attracted more and more attention from scholars.

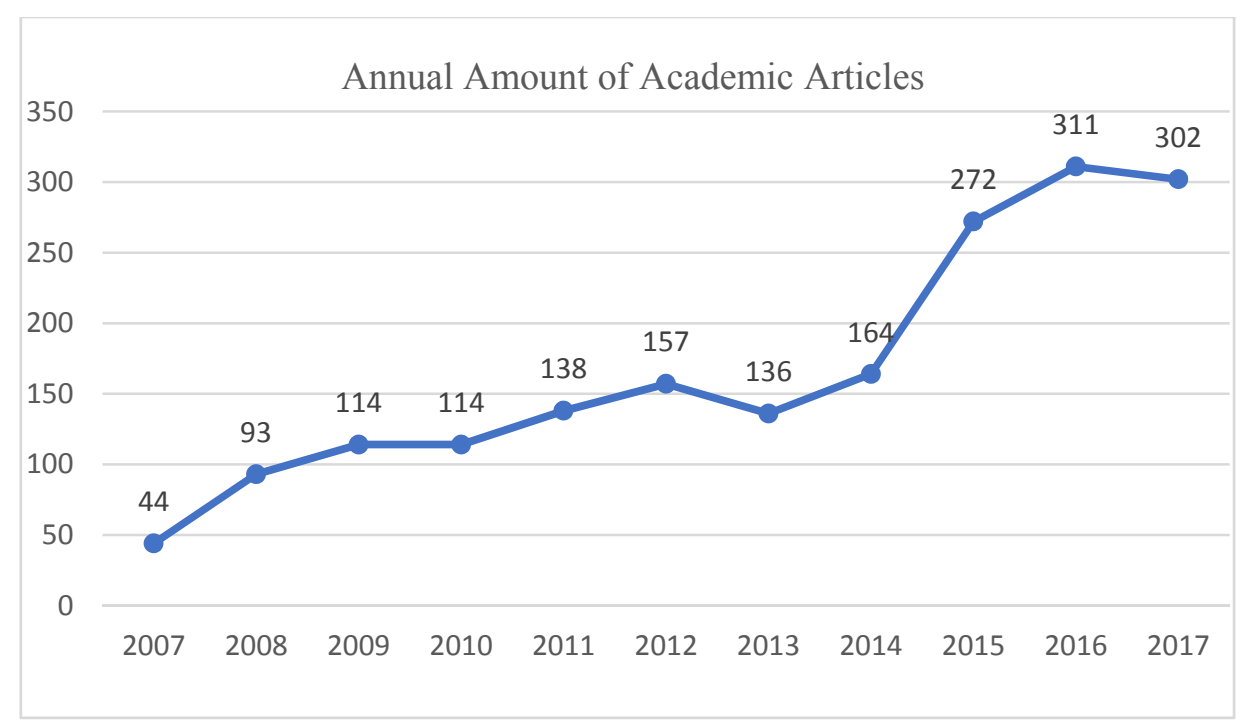

Fig 1. Longitudinal analysis of annual amount of articles

\subsection{High Productive Institutions and Scholars' Academic Influence}

The high productive institutions are widely distributed, the top two are the University of TexasAustin and the City University of New York in the United States, and the top ten are Arizona State University, University of California at Los Angeles, Texas A\&M university, the University of Colorado, University of Arizona and University of Illinois. These research institutions belong to the United States, and they have obvious advantages in the number of papers. Most of these high productive American institutions belong to the better ranked institutions of education in the United States, located in the coastal areas of the United States, with convenient transportation.

The prolific authors have similar quantities of papers, with the highest amount of writing from Hogue A from Lleida University in Spain and Schwartz M from the Oran Nimes Academy of Sciences, Israel, with 9 papers. The second is Bialystok E from York University, Canada, with a large number of third, respectively from the University of California at San Diego, University of Pennsylvania, Hebrew University Jerusalem, and China's University of Hong Kong. The number of papers is 5. Among the highly cited authors, they came from famous universities in the United States and Canada, such as Stanford University and York University. In addition, many high cited authors do not publish much, but they are cited heavily.

\subsection{Analysis of High Yield Periodicals and Highly Cited Periodicals}

From the top ten high-yield and highly cited periodicals, the first four are language classes. It can be seen that the research of international bilingual education has become increasingly mature, which is a major focus of international academic research. The following are cultural periodicals, psychological journals, teacher education journals, and curriculum publications, all of which are the focus of bilingual education research. Further analysis of highly cited journals, the top four are language periodicals, the fifth is psychology, sixth is pedagogy, and seventh is cultural periodicals. Among them, international journal of bilingual education and bilingualism was quoted the most, reaching 400 . We can see that most of the top ten periodicals in the high cited periodicals are closely related to bilingual education. The history of bilingual education in foreign countries is long, and the system is mature and complete in all aspects. In addition, some journals do not publish enough papers, but the number of cited papers is very high, such as applied psycholinguistics, review of educational research, tool quarterly, American educational research journal.

The top ten cited articles were ranked first by the Northwestern University Marion V, Blumenfeld HK and Kaushanskaya M in the Language Experience and Proficiency Questionnaire published in 
2007, which was quoted as high as 352 times. Secondly, the University of Minnesota Carlson SM, and the Bilingual experience and executive functioning in young children, published in 2008 by the Melt off AN of University of Washington, were cited as 346 times; The Bialystok E of York University in Canada and the Crack FIM of University of Toronto, Freedman M publish Bilingualism as a protection against the, and are quoted as 252 times. The Delaying the onset of Alzheimer disease Bilingualism as a form of cognitive reserve articles published in 2010 were cited 163 times. We can see that the research of neuroscience has a great influence in the study of international bilingual education. Of the top 10 cited articles, 4 have been published in the field of neuroscience. They have played a positive role in bilingual education and become a hot research field.

\section{Research Hotspots and Topics}

Through the study of keyword co-occurrence and cluster analysis, we find that the hot topics in bilingual education research focus on 12 aspects, the study of cross- linguistic, preschool children, cognitive reserve, two- way bilingual immersion, content and language integrated study, comprehensive education, South Africa, second language, teaching language, first and second language education, teacher identification, and immigrant student.

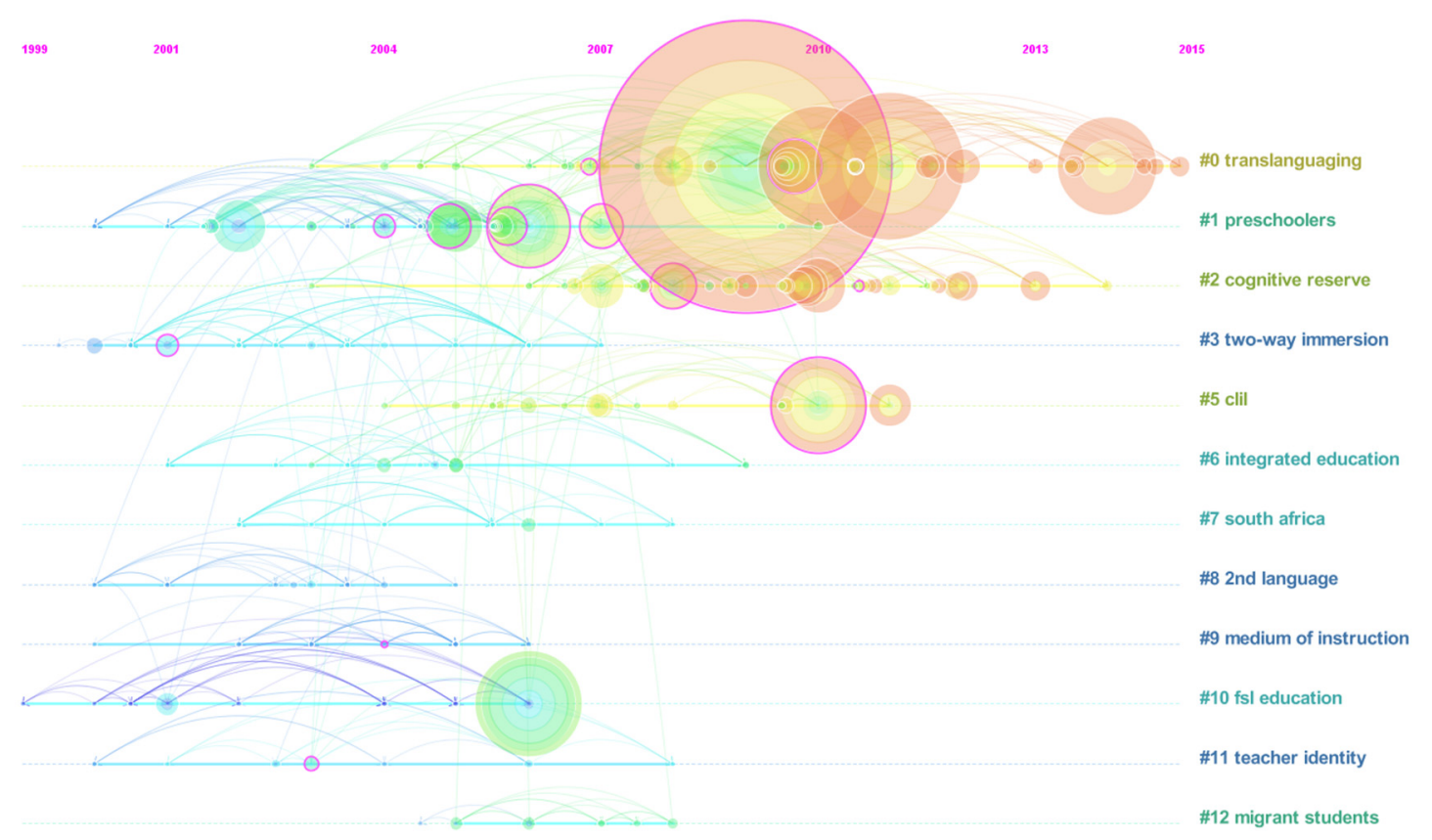

Fig 2. Timeline graph of keyword co-occurrence and clustering in bilingual education

In order to clarify the evolution of bilingual education research topics, we draw a timeline graph of keyword co-occurrence and clustering. The graph shows the research process of the hot topics in bilingual education from left to right in chronological order. The horizontal line represents the starting time of the research hotspot, and the time from left to right is constantly updated. The size of the bubble represents the number of results. The smaller bubbles represent the focus time of the hot spot, the larger the bubble is, and the greater the influence of the research on the hot spot in this period, and the arc network shows the influence of the research in the hot spot. It can be seen that there are some differences between the 12 hot spots of bilingual education in the research history and research density, and the cross-linguistic theme research is the most prominent in historical duration, the number of research results and the influence; The results of preschool children research were more than before 2007, which had great influence on this field. However, the output was very little and the influence was weak in 2007. The research on the topic of cognitive reserve has been stable in terms of the number and influence of the research results from 2003 to the present. In comparison, the research results of two-way bilingual immersion are few and weak in recent years. The major themes 
of \#3 to \#10 have also influenced the field before 2007, but the results are not much. The main results are the study of content and language integrated learning and the first and second language education research. There is no coherence in the historical dimensions of \#3 to \#12, or appear in the middle of a short period of time, or in the early stage of hot spots.

\section{Conclusions}

The United States and Canada occupy the dominant position in the field of bilingual education. The research and concern about bilingual education in China is mainly about bilingual teaching. "Bilingual Teaching" was popular in China since the beginning of twenty-first Century. In 2001, the Ministry of Education issued a number of opinions on strengthening the teaching quality of undergraduate teaching in Colleges and universities in 2001, and the educational circles in China began to appear [1]. Through the quantitative research on the important documents of international bilingual education, we can draw the following enlightenment:

First, bilingual education is of great value to the development of ethnic education and bilingual teaching in China. There are more than 200 sovereign states in the world, most of which are multiracial and multilingual. Economic development and technological progress have broken the geographical restrictions. In human history, language contact has never been as frequent as it is today. Therefore, bilingualism has become an international hot issue and has become an urgent problem faced by all countries [2]. Multicultural and multilingual countries need a language policy that takes into account not only the external language but also the internal language. In other words, we need to look outward towards a globalized world, and also focus on local complexity. Bilingual education enables students to prepare for contact with other countries and cultures, so that they can open the door to the world in the spirit of international understanding [3]. Bilingual education can maintain the diversity of world culture, cultivate students' positive attitudes towards different cultures and maintain national unity.

Second, the themes of bilingual education are widely distributed, and there are certain differences on study of history and density. The main research hotspots and stages of international bilingual education can be summarized into four types: (1) the core theme of long-term concern: the two research hotspots of cross language and cognitive reserve are the most prominent in terms of historical persistence and the number and influence of research results; (2) the core themes of the weakening in recent years: preschool children research, two-way bilingual immersion research, content and language integrated learning, first and second language education research; (3) stage research topics: comprehensive education research, South Africa research, second language research, teacher identification, immigrant students and other fields are relatively strong, and do not have historical inheritance. (4) National exclusive research: Immigration research and South African studies have national characteristics.

Third, from the perspective of bilingual education research, cross-linguistic research will become the focus of follow-up research. International journal of bilingual education and bilingualism, the mainstream Journal of cross-linguistic research, is the best in the international bilingual education research. Bilingual education, multiculturalism, teaching strategies and so on are the research topics that scholars both at home and abroad are paying close attention to. The world multiracial constitutes the world multicultural background. In today's pluralistic world, education will also be diversified. Bilingual education is the best way to diversify international cultural education in the future [4].

Finally, the research methods of international bilingual education are mainly qualitative research, involving educational narrative research, educational observation, meta-analysis, anthropology and ethnography. Quantitative research methods mainly involve psychological test, demographic statistics, questionnaire survey and systematic methodology. The study of bilingual education in China mainly follows the research methods of ethnic pedagogy, commonly used methods of comparative study, historical research, anthropology and sociology. At the same time, it draws lessons from the educational narrative, meta-analysis and psychological test of European and American countries, and advances the schools and communities in the ethnic areas of our China. In order to 
apply the research results and methods of bilingual education to the practice of the curriculum reform of bilingual education, a mixed study of the participatory action research and ethnographic investigation is carried out to carry out a mixed study of the educational policy, curriculum, teacher training and classroom teaching effect of bilingual education.

\section{References}

[1]. Liming $\mathrm{Yu}$, Elizabeth Yeoman. Bilingual Education-The Implications of the Canadian Immersion Education to the Bilingual Instruction in Chinese Universities [M].Beijing: Foreign language Teaching and Research Press, 2009.

[2]. Anderson Benedict. Imagined Communities: Reflections on the Origin and Spread of Nationalism [M].Verso, 2008.

[3]. Mejia, A.M.de (2015). Bilingual education in Colombia: Towards a Recognition of Languages, Cultures and Identities. Colombian Applied Linguistics Journal, 2015, 45 (2): 55.

[4]. Miguel Siuán, William Francis Mackey. Education and Bilingualism [M]. London: K. Page in association with Enesco, Paris, France, 1987. 